\title{
Comparison of hospital-wide and unit-specific cumulative antibiograms in hospital- and community-acquired infection
}

\author{
F. Lamoth · A. Wenger · G. Prod'hom • Y. Vallet • \\ C. Plüss-Suard $\cdot$ J. Bille $\cdot$ G. Zanetti
}

Received: 15 February 2010/Accepted: 25 May 2010/Published online: 16 June 2010

(C) Urban \& Vogel 2010

\begin{abstract}
Background Empirical antibacterial therapy in hospitals is usually guided by local epidemiologic features reflected by institutional cumulative antibiograms. We investigated additional information inferred by aggregating cumulative antibiograms by type of unit or according to the place of acquisition (i.e. community vs. hospital) of the bacteria. Materials and methods Antimicrobial susceptibility rates of selected pathogens were collected over a 4-year period
\end{abstract}

Electronic supplementary material The online version of this article (doi:10.1007/s15010-010-0033-0) contains supplementary material, which is available to authorized users.

F. Lamoth $(\bowtie) \cdot$ G. Zanetti

Infectious Diseases Service, Centre Hospitalier Universitaire

Vaudois and University of Lausanne, Rue du Bugnon 46,

1011 Lausanne, Switzerland

e-mail: Frederic.Lamoth@chuv.ch

\section{A. Wenger · G. Prod'hom - J. Bille}

Institute of Microbiology, Centre Hospitalier Universitaire

Vaudois and University of Lausanne, Lausanne, Switzerland

Y. Vallet

Informatics Institute, Centre Hospitalier Universitaire Vaudois and University of Lausanne, Lausanne, Switzerland

C. Plüss-Suard

Service of Pharmacy, Centre Hospitalier Universitaire Vaudois, Lausanne, Switzerland

C. Plüss-Suard

School of Pharmaceutical Sciences, University of Geneva and University of Lausanne, Geneva, Switzerland

G. Zanetti

Service of Hospital Preventive Medicine, Centre Hospitalier Universitaire Vaudois and University of Lausanne,

Lausanne, Switzerland in an university-affiliated hospital. Hospital-wide antibiograms were compared with those selected by type of unit and sampling time ( $<48$ or $>48 \mathrm{~h}$ after hospital admission). Results Strains isolated $>48 \mathrm{~h}$ after admission were less susceptible than those presumably arising from the community $(<48 \mathrm{~h})$. The comparison of units revealed significant differences among strains isolated $>48 \mathrm{~h}$ after admission. When compared to hospital-wide antibiograms, susceptibility rates were lower in the ICU and surgical units for Escherichia coli to amoxicillin-clavulanate, enterococci to penicillin, and Pseudomonas aeruginosa to anti-pseudomonal beta-lactams, and in medical units for Staphylococcus aureus to oxacillin. In contrast, few differences were observed among strains isolated within $48 \mathrm{~h}$ of admission. Conclusions Hospital-wide antibiograms reflect the susceptibility pattern for a specific unit with respect to community-acquired, but not to hospital-acquired strains. Antibiograms adjusted to these parameters may be useful in guiding the choice of empirical antibacterial therapy.

Keywords Antibiogram - Bacterial sensitivity tests . Hospital units · Nosocomial infections .

Antibiotic resistance

\section{Introduction}

Prompt initiation of effective antibiotic therapy has been associated with better survival in severe hospital- or community-acquired infections [1-3]. As microbiological documentation of infection is usually obtained within $24-48 \mathrm{~h}$ of symptom manifestation, the choice of empirical antibiotic therapy is crucial in the early management of severe infections. Such an empirical therapy should provide the optimized antibacterial spectrum that ensures coverage of the 
most common pathogens while avoiding the unnecessary selection of resistant bacteria [4, 5]. The use of hospital cumulative antibiograms (summary report of the percentages of susceptible strains of a given pathogen for the antibiotics routinely tested in an institution) to guide the choice of empirical antibiotic therapy has been identified as a key strategy to prevent and control the spread of resistant microorganisms in hospitals [4-6]. However, standardized guidelines for the generation of these reports are currently lacking [6]. Antimicrobial susceptibility patterns may vary between hospitals, and differences between units within a same institution have also been reported [7-12]. Similarly, differences have been observed between hospital- and community-acquired strains [13, 14].

The aim of this study was to investigate the additional information inferred from aggregating cumulative antibiograms by sampling time ( $<$ or $\geq 48 \mathrm{~h}$ from admission) and type of unit within one hospital.

\section{Materials and methods}

The study was carried out at the Centre Hospitalier Universitaire Vaudois (Lausanne, Switzerland), an 850-bed university-affiliated hospital. Data of in vitro susceptibility testing were collected from the microbiological laboratory over a 4-year period (2003-2006). The identification of bacterial species and in vitro susceptibility testing were performed according to the criteria of the American Society for Microbiology (ASM) [15] and the breakpoints definitions of the Clinical and Laboratory Standards Institute (CLSI, Wayne, PA) [16], respectively. Antibacterial susceptibility testing for the antibiotics most commonly used for a given microorganism were routinely performed for all potential pathogens isolated from any sample site. According to the microbiological laboratory's policy, this testing was not repeated when a microorganism was isolated more than once in the same patient within 4 days. Thereafter, it was performed every 4 days (if applicable). All isolates for whom antibacterial susceptibility testing had been performed were recorded in a computer-generated database. This system automatically excludes redundancies of strains (same microorganism with the same antibiotic susceptibility pattern detected in a separate isolate from a same patient). Using this database, cumulative antibiograms were retrospectively calculated for each year from 2003 to 2006 for the most frequent Gram-positive and -negative bacteria and the antibiotics most commonly prescribed in the institution. Some microorganisms were selected for their prevalence and the relevance of their susceptibility pattern in the choice of empirical antibacterial therapy: Escherichia coli (with respect to amoxicillin/clavulanate, ciprofloxacin, ceftriaxone), Pseudomonas aeruginosa (ceftazidime, cefepime, imipenem, meropenem, piperacillin/tazobactam ciprofloxacin), Staphylococcus aureus (oxacillin) and enterococci, including Enterococcus faecium/faecalis and other Enterococcus spp. (penicillin; of note, no enterococci nonsusceptible to vancomycin were documented during the study period). Antibiograms were extracted for the whole institution and for the following units: internal medicine, general surgery, adult intensive care (ICU) and medical paediatrics. A distinction was made between strains isolated $<48 \mathrm{~h}$ or $>48 \mathrm{~h}$ from admission (presumably community- and hospital-acquired, respectively) [17].

Strains were differentiated as "susceptible" and "nonsusceptible" (intermediate or resistant) according to the breakpoints definitions of the CLSI [16].

Susceptibility rates were compared between presumably community- and hospital-acquired strains and between the whole hospital and the units mentioned above. Differences between susceptibility rates aggregated by the site of sampling (blood cultures vs. cultures from any other site) were also investigated. Fisher's exact test was used to compare proportions. Two-sided $p$ values $<0.05$ were considered as statistically significant. Because of the large number of statistical comparisons, Bonferroni corrections for multiple tests were applied.

\section{Results}

Of the total number of strains analysed for the whole institution during the study period, 11,485 (44.5\% hospitalacquired) were $E$. coli strains, 4,638 (72.6\% hospital-acquired) were $P$. aeruginosa strains, 3,405 (65\% hospital-acquired) were enterococci strains and 8,008 (50.9\% hospitalacquired) were $S$. aureus strains.

Annual hospital-wide susceptibility rates of these pathogens remained stable over the 4-year period $(<5 \%$ variation over time for all of the antibiotics tested). Antibiograms calculated for the 4-year period were thus considered in further analyses.

For the whole institution, susceptibility rates were significantly lower for strains isolated $\geq 48 \mathrm{~h}$ from admission when compared to those isolated $<48 \mathrm{~h}$ ( $p$ value $<0.001$ for all pathogen/antibiotic combinations) (Table 1).

Among presumably community-acquired strains (isolated $<48 \mathrm{~h}$ from admission), antibiograms calculated for specific units displayed few differences when compared to those of the whole institution. The most relevant differences were observed for $P$. aeruginosa susceptibility to ciprofloxacin in medical and surgical units (64 vs. $80 \%$, $p<0.001$ and 62 vs. $80 \%, p=0.01$, respectively), and to imipenem in surgical units (71 vs. $87 \% ; p=0.01)$. In contrast, major differences between units were observed among hospital-acquired strains (Fig. 1a, b). P. aeruginosa 
Table 1 Comparison of the rates of susceptibility to selected antibiotics between presumably community- and hospitalacquired strains
Values are given as the number of susceptible strains/total number of strains, with the percentage of total in parenthesis

${ }^{\mathrm{a}} p$ value $<0.001$ for all comparisons between community- and hospitalacquired strains

\begin{tabular}{lll}
\hline $\begin{array}{l}\text { Microorganisms and } \\
\text { selected antibiotics to } \\
\text { which they showed susceptibility }\end{array}$ & $\begin{array}{l}\text { Community-acquired strains } \\
(<48 \mathrm{~h} \text { from admission) }\end{array}$ & $\begin{array}{l}\text { Hospital-acquired strains } \\
(>48 \mathrm{~h} \text { from admission })^{\mathrm{a}}\end{array}$ \\
\hline $\begin{array}{l}\text { Escherichia coli } \\
\text { Amoxicillin/clavulanate }\end{array}$ & $5,672 / 6,370(89)$ & $4,146 / 5,111(81)$ \\
$\quad$ Ceftriaxone & $6,301 / 6,372(99)$ & $4,940 / 5,113(97)$ \\
$\quad$ Ciprofloxacin & $5,860 / 6,348(92)$ & $4,342 / 5,091(85)$ \\
Pseudomonas aeruginosa & $1,179 / 1,269(93)$ & $2,807 / 3,368(83)$ \\
$\quad$ Cefepime & $1,148 / 1,269(90)$ & $2,638 / 3,368(78)$ \\
$\quad$ Ceftazidime & $1,100 / 1,269(87)$ & $2,389 / 3,368(71)$ \\
$\quad$ Imipenem & $1,157 / 1,269(91)$ & $2,703 / 3,367(80)$ \\
$\quad$ Meropenem & $1,215 / 1,269(96)$ & $3,039 / 3,368(90)$ \\
Piperacillin/tazobactam & $1,015 / 1,269(80)$ & $2,309 / 3,369(69)$ \\
$\quad$ Ciprofloxacin & & $3,439 / 4,077(84)$ \\
Staphylococcus aureus & $3,670 / 3,931(93)$ & $1,904 / 2,214(86)$ \\
$\quad$ Oxacillin & $1,151 / 1,192(97)$ & \\
Enterococci & & \\
$\quad$ Penicillin & & \\
\hline
\end{tabular}

susceptibility rates to most anti-pseudomonal beta-lactams were lower in surgical units and ICU than those calculated for the whole institution, while medical and paediatric units displayed similar or higher susceptibility rates (Fig. 1a). In addition, susceptibility of enterococci to penicillin was lower in ICU and surgical units (70 vs. $86 \%$ for the whole institution, $p<0.001)$ and susceptibility of $E$. coli to amoxicillin/clavulanate was lower in surgical units (70 vs. $81 \%, p<0.001)$. The susceptibility of $S$. aureus to oxacillin was lower in medical units (70 vs. $84 \%, p<0.001)$ (Fig. 1b).

The distinction according to the site of sampling (blood vs. any site) showed no differences above 5\% in susceptibility rates, with the exception of $P$. aeruginosa susceptibility to piperacillin/tazobactam (85 vs. $92 \%$ for blood cultures and cultures from any site, respectively, $p=0.015$ ) and ciprofloxacin (86 vs. $72 \%, p=0.001$ ), and enterococci susceptibility to penicillin (82 vs. $90 \%$, $p=0.002$ ).

\section{Discussion}

The results of our analysis revealed significant differences in antimicrobial susceptibility rates between presumably hospital- and community-acquired strains. These differences were particularly relevant for $P$. aeruginosa. The comparison between different units showed similar susceptibility rates among community-acquired strains, whereas important differences were observed among hospital-acquired strains, especially for $P$. aeruginosa and enterococci and with respect to the prevalence of methicillin-resistance in S. aureus (MRSA). However, stratification according to the site of sampling for cultures added little additional relevant information. These findings may have an impact on the choice of empirical antibiotic therapy, although it is limited to a subset of clinical situations and specific for the hospital where the data were collected.

Variability in the susceptibility rates between units in a same hospital has been reported previously [7-12]. Most differences were observed between the ICUs and nonICUs. However, little data are available on the comparison of cumulative antibiograms between wards other than ICUs and according to the sampling time in terms of being able to distinguish presumably nosocomial from communityacquired infections [7, 13, 14]. The study presented here proposes an integration of these easily available parameters. The potential impact of such a focused approach on the control of the emergence of resistance has been suggested in earlier studies [5, 18]. It should, however, be specified that the use of cumulative antibiograms aggregated according to ward or sampling time would only be possible if they are derived from a large enough number of isolates to reach statistical significance (provided that susceptibility rates remain stable over the time needed to obtain this number of isolates). Statistical significance is not enough, however, and unit- or sampling time-specific data should only be provided if they show clinically relevant gaps in antibiotic susceptibility. It may be the task of a hospital antibiotic committee to assess this relevance.

Resistance of $P$. aeruginosa to carbapenems is an important problem in ICUs and has been associated with exposure to this class of antibiotics [19-21]. Similarly, 
Fig. 1 Comparison of susceptibility rates between units within a same hospital for selected microorganism/ antibiotic combinations in hospital-acquired strains (culture drawn $>48 \mathrm{~h}$ from admission). $x$ axis Percentage of susceptible strains, black bars medical units, white bars surgical units, dark-grey bars intensive care units, light-grey bars pediatric units, black vertical lines susceptibility rate for the whole hospital. Numbers on the right $p$ value (Fisher's exact test with application of Bonferroni corrections for multiple tests) for the comparison between unitspecific and hospital-wide susceptibility rates, $N S$ not significant $(p>0.05)$. a Pseudomonas aeruginosa and cefepime, ceftazidime, ciprofloxacin, imipenem, meropenem and piperacillin/ tazobactam, b Staphylococcus aureus and oxacillin,

Escherichia coli and amoxicillin/clavulanate and ciprofloxacin, enterococci (Enterococcus faecalis/faecium and other enterococci species) and penicillin

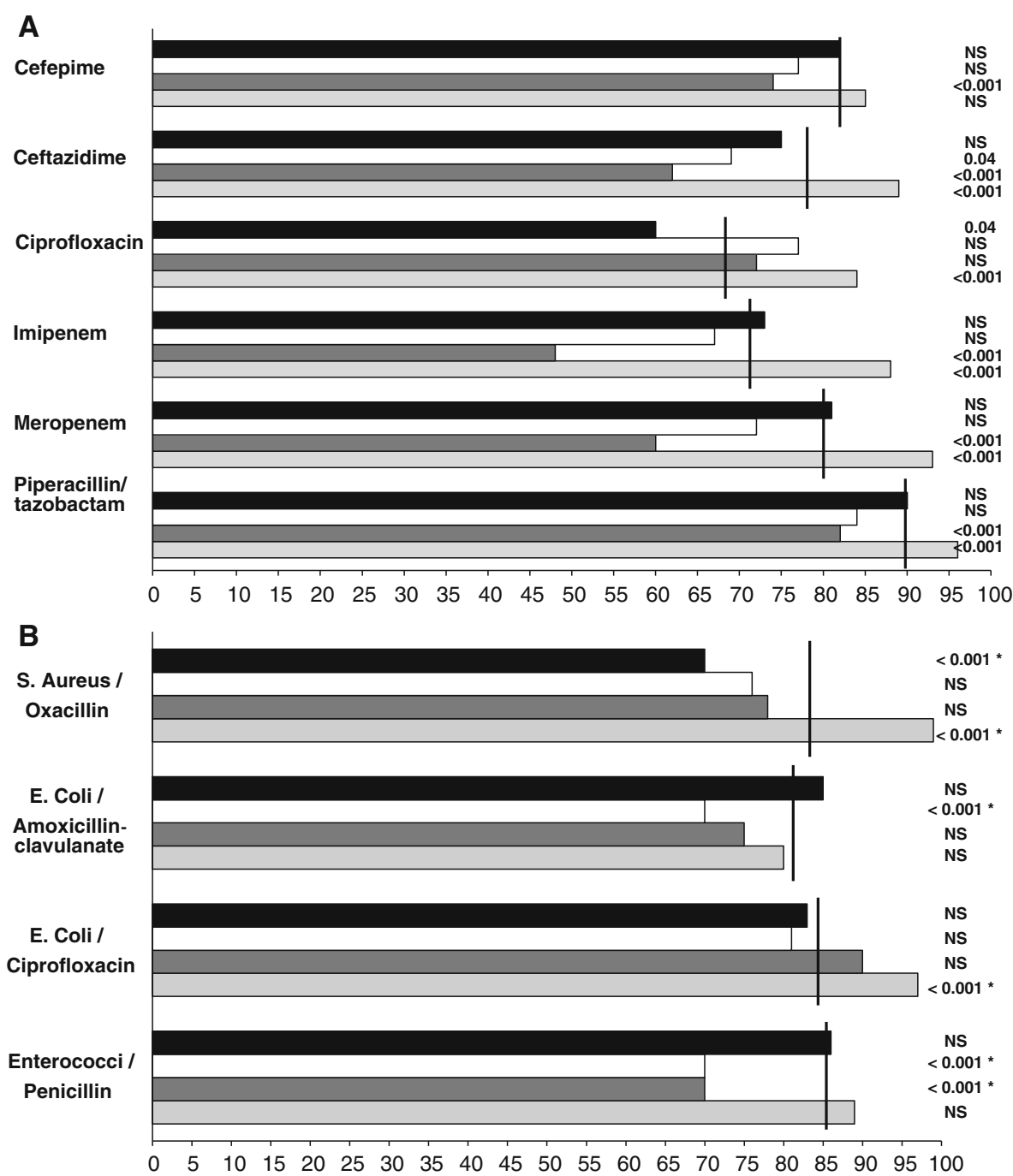

a low susceptibility rate of $P$. aeruginosa to carbapenems was observed among ICU isolates in our analysis. Data on antimicrobial consumption in our institution revealed that the use of carbapenems was particularly high in the ICU [22 defined daily dose (DDD)/100 bed-days for both imipenem and meropenem when compared to 4.5 for piperacillin/tazobactam, whereas carbapenems consumption in the whole institution was only 4 DDD/100 bed-days] [22]. The use of amoxicillin and amoxicillin/clavulanate in surgical units was also higher than that in medical units (22.4 and 14.3 DDD/100 bed-days, respectively), which may contribute to the lower susceptibility rate of enterococci to penicillin in the former setting.

This analysis has several limitations. First, it was conducted in a single centre. As such, these results may not be reproducible in other countries or institutions depending on potential differences in case-mix and epidemiologic characteristics. Differences observed in the prevalence of MRSA may particularly result from variations in the casemix between units or from possible cross-infections. In addition, individual risk factors for infections with resistant microorganisms, such as the duration of hospital stay, underlying diseases or previous antibacterial therapy, are not taken into account by such an approach based on aggregated data from the microbiology lab. However, the utility of cumulative antibiograms for guiding local empirical antibiotic strategies and preventing the emergence of resistance has been clearly established despite this intrinsic limitation [5]. Second, the 48-h cutoff was chosen according to the Centers for Disease Control standard definitions of nosocomial infections [17]. However, the incubation period of infection may vary according to the type of pathogen or the patient's underlying conditions, making the distinction between hospital- and communityacquired infections difficult to determine in some cases [17]. This may be of particular relevance for P. aeruginosa. Third, our data do not allow differentiating bacteria isolated in true infections from those considered to be colonizers or contaminants. However, such a distinction should not have resulted in significant differences in susceptibility 
rates. Finally, the repetition of antimicrobial susceptibility testing for a same strain isolated in a same patient may have been a source of bias despite the use of selection criteria to avoid such redundancies in our electronic database. One of the consequences is the impossibility to distinguish between primary or secondary acquired resistance.

In conclusion, the results of the analysis reported here suggest that hospital-wide cumulative antibiograms reflect the actual susceptibility pattern for a specific unit with regard to community-acquired infection, but not with regard to hospital-acquired infections. Institutional policies for the report of cumulative antibiograms should thus be adapted to these parameters that are easy to obtain. Such an approach may be useful in guiding the choice of empirical antibacterial therapy.

Conflict of interest statement None.

\section{References}

1. Harbarth S, Garbino J, Pugin J, Romand JA, Lew D, Pittet D. Inappropriate initial antimicrobial therapy and its effect on survival in a clinical trial of immunomodulating therapy for severe sepsis. Am J Med. 2003;115:529-35.

2. Kollef MH, Sherman G, Ward S, Fraser VJ. Inadequate antimicrobial treatment of infections: a risk factor for hospital mortality among critically ill patients. Chest. 1999;115:462-74.

3. Kumar A, Roberts D, Wood KE, Light B, Parrillo JE, Sharma S, et al. Duration of hypotension before initiation of effective antimicrobial therapy is the critical determinant of survival in human septic shock. Crit Care Med. 2006;34:1589-96.

4. Fridkin SK, Steward CD, Edwards JR, Pryor ER, McGowan JE Jr, Archibald LK, et al. Surveillance of antimicrobial use and antimicrobial resistance in United States hospitals: project ICARE phase 2. Project Intensive Care Antimicrobial Resistance Epidemiology (ICARE) hospitals. Clin Infect Dis. 1999;29:245-52.

5. Goldmann DA, Weinstein RA, Wenzel RP, Tablan OC, Duma RJ, Gaynes RP, et al. Strategies to prevent and control the emergence and spread of antimicrobial-resistant microorganisms in hospitals. A challenge to hospital leadership. JAMA. 1996; 275:234-40.

6. Fridkin SK, Edwards JR, Tenover FC, Gaynes RP, McGowan JE Jr. Antimicrobial resistance prevalence rates in hospital antibiograms reflect prevalence rates among pathogens associated with hospital-acquired infections. Clin Infect Dis. 2001;33:324-30.

7. Binkley S, Fishman NO, LaRosa LA, Marr AM, Nachamkin I, Wordell D, et al. Comparison of unit-specific and hospital-wide antibiograms: potential implications for selection of empirical antimicrobial therapy. Infect Control Hosp Epidemiol. 2006; 27:682-7.
8. Bryce EA, Smith JA. Focused microbiological surveillance and gram-negative beta-lactamase-mediated resistance in an intensive care unit. Infect Control Hosp Epidemiol. 1995;16:331-4.

9. Kaufman D, Haas CE, Edinger R, Hollick G. Antibiotic susceptibility in the surgical intensive care unit compared with the hospital-wide antibiogram. Arch Surg. 1998;133:1041-5.

10. Namias N, Samiian L, Nino D, Shirazi E, O’Neill K, Kett DH, et al. Incidence and susceptibility of pathogenic bacteria vary between intensive care units within a single hospital: implications for empiric antibiotic strategies. J Trauma. 2000;49:638-45.

11. Pierson CL, Friedman BA. Comparison of susceptibility to betalactam antimicrobial agents among bacteria isolated from intensive care units. Diagn Microbiol Infect Dis. 1992;15:19S-30S.

12. Stratton CW, Ratner H, Johnston PE, Schaffner W. Focused microbiologic surveillance by specific hospital unit as a sensitive means of defining antimicrobial resistance problems. Diagn Microbiol Infect Dis. 1992;15:11S-8S.

13. Archibald L, Phillips L, Monnet D, McGowan JE Jr, Tenover F, Gaynes R. Antimicrobial resistance in isolates from inpatients and outpatients in the United States: increasing importance of the intensive care unit. Clin Infect Dis. 1992;24:211-5.

14. Harbarth S, Rohner P, Safran E, Garbino J, Auckenthaler R, Pittet D. Resistance to amikacin and gentamicin among Gram-negative bloodstream isolates in a university hospital between 1989 and 1994. Clin Microbiol Infect. 1998;4:199-204.

15. Jorgensen JH. Antibacterial agents and susceptibility test methods. In: Murray PR, Baron EJ, Jorgensen JH, Pfaller MA, Yolken RH (eds) Clinical microbiology, vol. 1. 8th edn. Washington: ASM Press; 2003. p. 1037-213.

16. Clinical and Laboratory Standards Institute. Performance standards for antimicrobial susceptibility testing. 17th Informational supplement. CLSI, 2007 document M100-S17. Clinical and Laboratory Standards Institute, Wayne. 2007.

17. Horan TC, Andrus M, Dudeck MA. CDC/NHSN surveillance definition of health care-associated infection and criteria for specific types of infections in the acute care setting. Am J Infect Control. 2008;36:309-32.

18. Stratton CW, Ratner H, Johnston PE, Schaffner W. Focused microbiologic surveillance by specific hospital unit: practical application and clinical utility. Clin Ther. 1993;15:12-20.

19. Carmeli Y, Troillet N, Eliopoulos GM, Samore MH. Emergence of antibiotic-resistant Pseudomonas aeruginosa: comparison of risks associated with different antipseudomonal agents. Antimicrob Agents Chemother. 1999;43:1379-82.

20. El Amari EB, Chamot E, Auckenthaler R, Pechere JC, Van Delden C. Influence of previous exposure to antibiotic therapy on the susceptibility pattern of Pseudomonas aeruginosa bacteremic isolates. Clin Infect Dis. 2001;33:1859-64.

21. Meyer E, Schwab F, Gastmeier P, Rueden H, Daschner FD. Surveillance of antimicrobial use and antimicrobial resistance in German intensive care units (SARI): a summary of the data from 2001 through 2004. Infection. 2006;34:303-9.

22. WHO Collaborating Centre for Drug Statistics Methodology. Guidelines for ATC classification and DDD assignment 2007. Oslo. 2007. Available at: http://www.whocc.no/atcddd/. 